\title{
A bridge between evidence-based laboratory diagnostics and research in neuroimmunology: why standardizations and guidelines matter
}

\author{
Diego Franciotta ${ }^{1}$ - Antonio Uccelli ${ }^{2,3,4}$ - Under the auspices of the Italian Association of \\ Neuroimmunology (AINI)
}

(C) Springer-Verlag Italia S.r.1. 2017

Keywords Autoantibodies · Anti-myelin oligodendrocyte glycoprotein antibodies $\cdot$ National healthcare system ·

Neurological autoimmune diseases $\cdot$ Statistics

\section{"The virtue of a thing consists in the fact that it is in the proper condition for the fulfilment of its nature or its purpose"}

Plato, The Republic (380 BC)

As the Latin root suggests, the idea of a "supplement" conveys the need, or the opportunity at least, of providing something that currently lacks. Therefore we cannot help but explain the compelling and deep reasons underlying the present supplementum, which was born within the Italian Association of Neuroimmunology (AINI), and is now finding its natural landing place on the Journal of the Italian Society of Neurology (SIN) for a wider diffusion.

The gist of our work comes from a simple concept, that "patients deserve that [their biological samples have to be] analyzed in a manner that they will yield the most informative

Diego Franciotta

diego.franciotta@mondino.it

1 Laboratory of Neuroimmunology, IRCCS, C. Mondino National Neurological Institute, I-27100 Pavia, Italy

2 Department of Neurosciences, Rehabilitation, Ophthalmology, Genetics, Maternal and Child Health Unit, University of Genoa, Genoa, Italy

3 IRCCS Azienda Ospedaliera Universitaria San Martino-IST, Genoa, Italy

4 Center of Excellence for Biomedical Research (CEBR), University of Genoa, Genoa, Italy results," as stated in a consensus on the cerebrospinal fluid (CSF) analysis [1]. We believe that this principle should be extended to all laboratory tests. The requirements for achieving that goal, and assuring accuracy and reliability of test results, include: (a) standardization of methods, (b) clinicolaboratory guidelines, and (c) periodic monitoring of the laboratory analytical performance by external quality controls. Over the years, AINI has promoted these three points by exploiting consensus-based programs for procedure and method standardization.

An important impulse to the revitalization of neuroimmunological diagnostics has come from the discovery of autoantibodies to surface membrane neuronal antigens and the availability of tests for their detection. Such antibody reactivities are associated with particular forms of "autoimmune encephalitis," potentially treatable diseases representing an expanding area in neurology [2, 3] (see the guideline article in this supplementum). Table 1 shows a list of antigens targeted by these new autoantibodies together with other most common antigens involved in autoantibody-related neurological diseases.

The fact that standardization is increasingly becoming a mandatory procedure not only in neuroimmunological diagnostics but also in research fields can be no longer overlooked. In recent years, growing attention has been paid to the factors that can bias research products and their interpretations. These factors range from the psychological theory of self-deceiving [4], which can be summarized by quoting Richard Feynman in his 1974 commencement address at Caltech: "the first principle [for scientific integrity] is that you must not fool yourself, and you are the easiest person to fool," to the misuse and misinterpretation of statistics, which possibly supports a trend towards the publication of research findings claimed solely on their achievement of formal statistical significance, often refuted by subsequent evidence [5]. The misuse of $p$-values is 
Table 1 List of the main antigens associated with the most common autoantibody-related neurological diseases

\begin{tabular}{lll}
\hline Antigens & Neurological diseases & Methods of detection \\
\hline NMDA-R & NMDA-R encephalitis & CBA, IHC \\
Lgi1 & Limbic encephalitis, FBDS & CBA, IHC \\
Caspr2 & Neuromyotonia, Morvan syndrome & CBA, IHC \\
Hu (ANNA-1) & Encephalomyelitis, limbic encephalitis, subacute sensory & IIF/IHC, dot/line blot \\
& neuronopathy, brainstem, encephalitis, chronic intestinal & \\
Yo (PCA-1) & Subacute cerebellar degeneration & IIF/IHC, dot/line blot \\
Ri (ANNA-2) & Opsoclonus-myoclonus & IIF/IHC, dot/line blot \\
Tr & Subacute cerebellar degeneration & IIF/IHC, dot/line blot \\
Amphiphysin & Stiff person syndrome, encephalomyelitis & IIF/IHC, dot/line blot \\
GAD & Stiff person syndrome; cerebellar ataxia & IIF/IHC, ELISA/RIPA \\
CV2/CRMP5 & Cerebellar degeneration, encephalomyelitis, limbic encephalitis & IIF/IHC, dot/line blot \\
Ma2 & Limbic encephalitis & IIF/IHC, dot/line blot \\
VGCC & Lambert-Eaton myasthenic syndrome & RIPA \\
ACh-R & Myasthenia gravis & RIPA \\
MuSK & Myasthenia gravis & RIPA \\
Aquaporin-4 & NMOSD & CBA \\
MOG & NMOSD, ADEM & CBA \\
MAG & IgM paraproteinemic neuropathy & ELISA \\
GM1, GM2 & IgM-associated multifocal motor neuropathy & Dot/line blot, ELISA, TLC \\
GQ1b, GT1a & Fisher syndrome, Bickerstaff syndrome & Dot/line blot, ELISA, TLC \\
\hline
\end{tabular}

$N M D A-R$, N-methyl-D-aspartate receptor; $C B A$, cell-based assay; $I H C$, immunohistochemistry; $I I F$, indirect immunofluorescence Lgi1, leucine-rich, glioma inactivated 1; FBDS, faciobrachial dystonic seizures; Caspr2, contactin associated protein-like 2; $A N N A$, anti-neuronal nuclei antibody; $P C A$, Purkinje cell antibody; $G A D$, glutamic acid decarboxylase; ELISA, enzyme-linked immunosorbent assay; CRMP5, collapsin response mediator protein 5; VGCC, voltage-gated calcium channel; RIPA, radioimmunoprecipitation assay; $A C h-R$, acetylcholine receptor; $M u S K$, muscle specific kinase; $M O G$, myelin oligodendrocyte glycoprotein; $N M O S D$, neuromyelitis optica spectrum disorders; $A D E M$, acute disseminated encephalomyelitis; $M A G$, myelin-associated glycoprotein; $T L C$, thin layer chromatography exemplary of statistics misinterpretation. $P$-values only measure whether observed findings are attributable to chance but cannot answer per se the original and real question of whether the leading hypothesis is correct. The odds that the hypothesis is correct depend on the strength of the results and the plausibility of the hypothesis in the first place [5]. Too many studies "chase small effects hidden in noisy data" [5]. On the contrary, it seems not trivial to remind the following sentence by the philosopher of science Karl Popper, "It is the most characteristic feature of the scientific method that scientists will do everything they can in order to criticize and test the theory in question" [6]. Finding a solution to these shortcomings entails efforts to join groups and resources ("team of rivals" and "crowdsourcing research" are the key words) and to promote cross-lab replications [4] that is substantially to promote standardizations. The National Institutes of Health have recognized the status quo and outlined a plan to thoroughly address these issues [7].

\section{Standardizations and guidelines}

To standardize means to produce documents providing requirements, specifications, and guidelines which, in the case of laboratory diagnostics, should guarantee that methods and procedures yield accurate test results. The National Committee for Clinical Laboratory Standards represents the best model in this field. The committee regulates and monitors the activities of laboratories in the USA, promotes these activities worldwide ("globalization program"), and produces standards for analytical assays.

Since 2000, AINI has been promoting consensus-based programs for the standardization of procedures and methods in neuroimmunology and guidelines aimed at addressing how to choose laboratory tests within the right clinical contexts. As a matter of fact, requiring a test within a patients' population in which the suspected disease is "sufficiently prevalent" maximizes positive predictive values and reduces the number of 
false positive results, cutting down unnecessary tests and costs. It is intuitive that whatever good laboratory test (e.g., with sensitivity and specificity of 95\%), if applied to subjects whose probability of having a disease is very low, will yield almost only false positive results $(5 \%)$. In statistics, this fundamental concept is expressed by Bayes' theorem, which defines the logical transition between laboratory data and their use within the right clinical contexts, taking into account the centrality of disease prevalence.

AINI Centers have been repeatedly invited to participate in questionnaire-based surveys on their clinical and laboratory activities. The latest data on clinical activities (2014) show that the Centers manage about 14,000 patients with multiple sclerosis (MS), 1400 patients with myasthenia gravis, 2400 patients with dysimmune peripheral neuropathies, 120 patients with neuromyelitis optica spectrum disorders, 50 patients with paraneoplastic neurological syndromes, and 100 patients with autoimmune encephalitis. A real gold mine for collaborative studies.

The questionnaires also requested information on: (a) types of laboratory tests, (b) number of tests performed annually, (c) methodologies, and (d) participations in external quality control schemes.

The Centers have been dealing with inter-laboratory variability in procedures and methods, trying to uniform them. The documents in this supplementum derive from the latest update on standardizations and guidelines in neuroimmunology modified and approved in 2016 (AINI Congress in Lecce, Italy). Selected AINI centers with demonstrated area-specific expertise drafted the first versions of the documents then submitted to the consensus process through questionnaire-based surveys, internet contacts, and discussions at standardization-dedicated AINI workshops. The documents, which will be periodically updated, report essential disorder-related clinical information, tests' indications and limits, instructions for result interpretation, and agreed laboratory protocols.

\section{Normative landscape and guideline application in routine diagnostics}

Many reports recommend expertise in neuroimmunology diagnostic tests and their implementation in specialized laboratories [3, 8-11]. Nevertheless, laboratory medicine of autoimmune diseases is being increasingly conducted in large generalist laboratories that, differently from small specialized laboratories, can comply with the certification rules requested by regional agencies and national healthcare system in Italy. The availability of commercial tests certified for diagnostic use is favoring this critical transition from small to large laboratories.
Especially for some disorders, such as paraneoplastic neurological syndromes and autoimmune encephalitis, commercial tests can perform suboptimally $[12,13]$, and more complex inhouse tests are needed to decipher difficult clinical cases or dubious laboratory results. Moreover, in large laboratories the lack of expertise in neuroimmunological diagnostics, which includes unawareness of method limitations and scarce neurological culture, can result in possible inefficiency of the service and confusion for clinical neurologists.

European models promote few specialized centers that assure high-quality and cost-effective diagnostics to a network of neurological clinics also involved in multicenter research studies. In Italy, we could approximate these models through joint efforts by SIN and AINI. Meanwhile, it is mandatory to have a network of specialized laboratories to support all the other laboratories. As a whole, networking and standardizations mean rationalization of test requests, high-quality outputs, less misdiagnosis, less wrong therapies, and thus the best patient care with cost cuts for the national healthcare system.

\section{Future perspectives}

Anti-myelin oligodendrocyte glycoprotein (MOG) antibodies are waiting for standardization and guidelines. The history of these antibodies is long and winding. For about 20 years they were associated with MS, but the demonstration of the conformational nature of MOG binding sites [14] confirmed the suspect that the denaturing methods used for their detection until then were unfit. Already in 2005, a European quality control scheme had shown between-center discordances (personal communication). Ten years after, using a cell-based assay (CBA) able to detect conformational anti-MOG antibodies and testing sera of a large series of neurological patients, Waters and Colleagues showed that such antibodies associated with non-MS inflammatory neurological diseases [15].

At present, two European groups have proposed two live CBAs for anti-MOG antibody detection, which differ for the use of secondary antibodies, namely either anti-human total IgG [16] or anti-human IgG1 [15]. The methods likely differ in analytical sensitivity and specificity, which warrant appropriate studies. A commercial fixed CBA is available (Euroimmun, Lübeck, Germany). Notwithstanding that fixation could alter the MOG conformational binding sites, with loss of analytical sensitivity, the method showed good agreement with live CBA [17].

Anti-MOG antibodies, whose pathogenic meaning is still partially unknown, are detected in acute disseminated encephalomyelitis, and in inflammatory CNS demyelination manifesting phenotypes homologous to those typical of aquaporin-4-related neuromyelitis optica spectrum disorders 
[18]. The intrathecal production of anti-MOG antibody is uncommon, so they are detectable more consistently in serum than in the CSF [19]. Standardization and guidelines for antiMOG antibodies will be the forthcoming AINI task.

In conclusion, the exemplary history of anti-MOG antibodies further emphasizes the unmet need for shared guidelines with stringent methodological criteria to apply in neuroimmunology diagnostic tests and in the correlated research fields. This supplementum, resulting from the work of a whole Italian scientific community, ambitiously aims at fulfilling this need.

Acknowledgements DF is the coordinator of the AINI "standardization of procedures and methods in neuroimmunology" committee. AU is the AINI president. We thank Matteo Gastaldi and Silvia Franciotta for critical reading of the manuscript, the Journal's senior editor Barbara Pedrotti for her impeccable assistance, Gianvito Martino who sew the seed of this plant, and Francesco Lolli who made the plant grow lush.

\section{Compliance with ethical standards}

Conflict of interest The authors declare that they have no conflict of interest.

\section{References}

1. Freedman MS, Thompson EJ, Deisenhammer F et al (2005) Recommended standard of cerebrospinal fluid analysis in the diagnosis of multiple sclerosis: a consensus statement. Arch Neurol 62: 865-870

2. Irani SR, Gelfand JM, Al-Diwani A, Vincent A (2014) Cell-surface central nervous system autoantibodies: clinical relevance and emerging paradigms. Ann Neurol 76:168-184

3. Graus F, Titulaer M, Balu R et al (2016) A clinical approach to diagnosis of autoimmune encephalitis. Lancet Neurol 15:391-404

4. Nuzzo R (2015) Fooling ourselves. Nature 256:182-185

5. Ioannidis JPA (2005) Why most published research findings are false. PLoS Med 2:e124
6. Popper KR (1940) What is dialectic? Mind 49:403-426

7. Collins FS, Tabak L (2014) NIH plans to enhance reproducibility. Nature 505:612-613

8. Andersson M, Alvarez-Cermeño J, Bernardi G et al (1994) Cerebrospinal fluid in the diagnosis of multiple sclerosis: a consensus report. J Neurol Neurosurg Psychiatry 57:897-902

9. Willison HJ, Veitch J, Swan AV et al (1999) Inter-laboratory validation of an ELISA for the determination of serum anti-ganglioside antibodies. Eur J Neurol 6:71-77

10. Graus F, Delattre JY, Antoine JC et al (2004) Recommended diagnostic criteria for paraneoplastic neurological syndromes. J Neurol Neurosurg Psychiatry 75:1135-1140

11. Zuliani L, Graus F, Giometto B, Bien CG, Vincent A (2012) Central nervous system neuronal surface antibody associated syndromes: review and guidelines for recognition. J Neurol Neurosurg Psychiatry 83:638-645

12. Sabater L, Saiz A, Dalmau J, Graus F (2016) Pitfalls in the detection of CV2 (CRMP5) antibodies. J Neuroimmunol 290:80-83

13. Gastaldi M, Thouin A, Franciotta D, Vincent A (2017) Pitfalls in the detection of N-methyl-d-aspartate-receptor (NMDA-R) antibodies. Clin Biochem 50:354-355

14. Mayer MC, Breithaupt C, Reindl M et al (2013) Distinction and temporal stability of conformational epitopes on myelin oligodendrocyte glycoprotein recognized by patients with different inflammatory central nervous system diseases. J Immunol 191:3594-3604

15. Waters P, Woodhall M, O'Connor KC et al (2015) MOG cell-based assay detects non-MS patients with inflammatory neurologic disease. Neurol Neuroimmunol Neuroinflamm 2:e89

16. Di Pauli F, Mader S, Rostasy K et al (2011) Temporal dynamics of anti-MOG antibodies in CNS demyelinating diseases. Clin Immunol 138:247-254

17. Jarius S, Ruprecht K, Kleiter I et al (2016) MOG-IgG in NMO and related disorders: a multicenter study of 50 patients. Part 1: frequency, syndrome specificity, influence of disease activity, long-term course, association with AQP4-IgG, and origin. J Neuroinflamm 13:279

18. Reindl M, Di Pauli F, Rostásy K, Berger T (2013) The spectrum of MOG autoantibody-associated demyelinating diseases. Nat Rev Neurol 9:455-461

19. Höftberger R, Sepulveda M, Armangue T et al (2015) Antibodies to MOG and AQP4 in adults with neuromyelitis optica and suspected limited forms of the disease. Mult Scler 21:866-874 